\title{
Beiträge zur Kenntnis der Lippenplatte des Koreanischen Eichhorns (Eutamias Asiaticus Uthensis, Pallas).
}

\author{
Von
}

\section{Itaru Shibata und Masaru Utida.}

(Aus dem Anatomischen Institut der Medizinischen Akademie zu Keijo.)

Im Jahre 1924 beschrieb S. Schumacher bei Sciurus vulgaris L. eine platte, ovale, dem Mundwinkel näher gelegene, von der Umgebung gut abgegrenzte Hervorragung der Schleimhaut der Oberlippe und bezeichnete sie als "Lippenplatte." Nach seiner Angabe ist die Platte widerstandsfähiger als der umgebende Schleimhautteil und entspricht ihre Lage dem Diastem zwischen dem Schneide- und dem vordersten Mahlzahne.

Seit einigen Jahren haben wir die Lippenplatte auch bei Eutamias asiaticus uthensis (Pallas) beobachtet, wie beim obengenannten Tiere. Und zwar wiesen wir hier nach mikroskopischer Untersuchung einen hyalinen Knorpel in ihrem Innern nach. Solche Tatsache scheint uns doch bis jetzt noch nicht berichtet worden zu sein. Daher teilen wir eine kurze Darlegung unserer eigenen Resultate über die Platte mit.

Das benutzte Material besteht aus 6 Exemplaren von Eutamias asiaticus uthensis (P.), dessen Körperlänge zwischen dem Rüssel und dem After I 3-I4 $\mathrm{cm}$ beträgt, und zeigt äusserlich keine merkwürdigen Anomalien.

In allen Fällen gibt es hier symmetrisch eine von der umgebenden Schleimhautfläche sich etwas abhebende, kleine, platte, ovale Hervorragung auf der Oberlippenschleimhaut. Dies ist die sogenannte Lippenplatte von Schumacher. Ihr Durchmesser hat 4-4.5 mm Länge und $2 \mathrm{~mm}$ Breite, die Längsachse neigt etwas von dorso-rostral zu ventro-kaudal. Die Entfernung des vordersten Plattenendes vom vorderen Gingivarand zwischen den beiden Schneidezähnen sowie auch die des hintersten vom Mundwinkel ist respektiv 3-4 $\mathrm{mm}$ und $4 \mathrm{~mm}$. In der ganzen Mundhöhle nimmt die Platte ihre Lage gerade lateral von der Gaumenpapille entsprechend dem Diastem zwischen dem 
Schneide- und dem vordersten Mahlzahne ein, jedoch ist die Platte kürzer als die Breite des letzteren und füllt nur dessen einen Teil. (Fig. I).

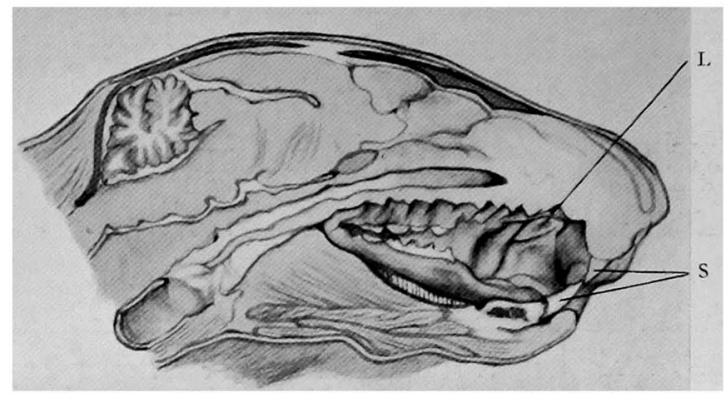

Fig. I. Sagittalschnitt eines Kopfes von Eutamias asiaticus uthensis (P.), von innen gesehen. Halbschematisch dargestellt.

$\mathrm{L}=$ Lippenplatte, $\mathrm{S}=$ Schneidezähnc.

Die Zunge ist grösstenteils abgeschnitten.

Abgesehen von der vorderen Umgebung, ist die Lippenplatte ebenso wie dorsal, auch ventral und kaudal von einer kontinuierlichen Grenzfurche umgeben, die die Platte von der übrigen Schleimhaut ausgeprägt abgrenzt und noch besonders tiefer an der Dorsalrinne ist. Wenn wir das Resultat von Schumacher und seiner Abbildungen berücksichtigen, so bemerken wir, dass unser eigener Fall von den seinigen folgendermassen abweicht: es gibt dicht kaudal vom kaudalsten Ende der Platte kein gut gebildetes, dreieckiges, seichtes Grübchen, und die obengenannte Grenzfurche wird an der rostralen Umgebung unterbrochen durch die direkte Fortsetzung der Plattenoberfläche mit der flachen Schleimhauterhebung, welche vor der Platte liegen und zu der entlang der Ventralseite der ventralen Grenzrinne verlaufenden niedrigen ventralen Grenzleiste übergehen. Kein Verwachsungswulst von Schumacher kommt hier vor. Doch schliesst sich an die dicht kaudale Umgebung der Lippenplatte, durch die Grenzrinne abgegrenzt, eine sehr deutliche spindelförmige, fast vertikal von oben rostral nach unten kaudal verlaufende Falte, deren unteres Ende weit in die Backentasche hinein läuft. Diese ist ein Teil einer blätterförmigen, frontal gerichteten Falte, die von der lateralen Taschenwand aus in der Taschenhöhle hervorragt und ventralwärts almählich niedriger wird. Die Oberfläche der Platte vertieft sich rinnenartig in seichtem Grade entlang der Plattenlängsachse.

Zur mikroskopischen Untersuchung wird die Lippenplatte mit den übrigen Lippenteilen nach Fixierung mit 10\% iger Formalinlösung oder Alkohol in Serien gefertigt, die je nach Bedarf zur Übersicht des Knorpels oder detaillierter Untersuchung der Gewebsbestandteile resp. in 30/ dicke Zelloidin- oder 5-10" dicke Paraffin-schnitte zerlegt, und mit Eosin-Hämatoxylin oder unter Umständen mit Resorcin-Fuchsin nach Weigert, van Gieson'scher Lösung und Mallory'scher Lösung gefärbt werden.

Nach Erforschung dieser Präparate (Fig. 2) ist das Epithel der Lippenplatte, ebenso wie das der üblichen Mundschleimhautepithelien, geschichtetes 


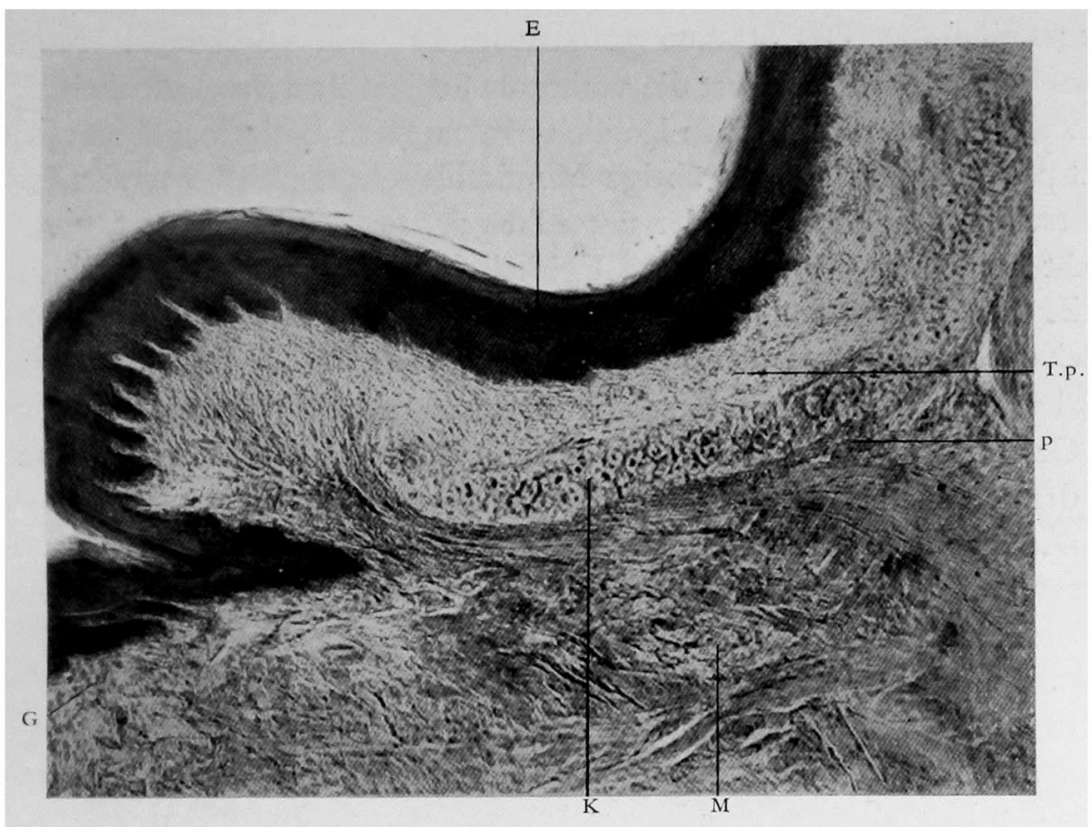

Fig. 2. Querschnitt der Lippenplatte von Eutamias asiaticus uthensis (P.). Eosin-Haematoxylinfärbung. Schnittdicke jo $\mu$.

$\mathrm{E}=$ Epithel, $\mathrm{K}:=$ Knorpelgewebe, T.p.=Tunica propria, $\mathrm{M}=$ quergestreifte Muskelfaser, $\mathrm{G}=\operatorname{der}$ Grenzleiste entsprechender Schleimhautteil, $\mathrm{P}=$ Perichondrium.

Plattenepithel. Seine Gesamtdicke ist in der Mitte am stärksten und beträgt etwa 90-105', doch wird sie nach der Umgebung zu so dünn, dass sie nur I/3-1/2 so dick wie die Mitte ist-sie beläuft sich an der Dorsal- und der Ventralgrenzrinne auf 30-40\%, und an der dieselben von dorsal begrenzenden Schleimhaut $45 \%$. Die Grenzleiste entspricht dem unbehaarten Teil, der zwischen der Ventralgrenzrinne und dem behaarten Teil der Innenfläche der Oberlippe liegt, und ist hier das Epithel in der Regel gleich dick wie das der Plattenmitte. Aber das Epithel der Leiste vermindert sich plötzlich zu i $5 \%$ nach dem behaarten Teil und setzt sich an der Epidermis fort.

An der Oberfläche des Lippenplattenepithels befindet sich die sogenannte „Verhornte Schicht " von Schumacher. Diese Schicht ist in der Plattenmitte I 8-24"' dick, besteht aus geschichteten, dicht gedrängten und deutlich abgeplatteten Zellen mit einem rudimentären Kern und erinnert an die Nagelplatte, wie schon Schumacher angegeben hat. In einem Ausnahmefall enthält die Zelle noch einen piknotischen länglich-ovalen Kern. Am tiefsten der Schicht ordnen sich die spindelförmigen, mit Eosin relativ stark gefärbten, mit einem piknotischen länglich-ovalen Kerne versehenen Zellen nebeneinander etwa in 
einer Reihe an. Das Stratum granulosum ist nicht vorhanden. Nach der Dorsalgrenzrinne hin ändert die verhornte Schicht ihre Beschaffenheit, indem die Zellen einen piknotischen länglich-ovalen Kern besitzen. Diese Schicht geht peripheriewärts in das übrige Mundschleimhautepithel über. Dennoch unterscheidet sie sich von der unter ihr gelegenen Epithelschicht, und noch macht sie einen der verhornten Epithelschicht ähnlichen Eindruck. Ihre Dicke beträgt $7-9 \mu$, und das Dickenverhältnis zu der Plattenmitte ist ungefähr gleich wie das der gesamten Epitheldicke zwischen beiden Teilen.

Die verhornte Schicht setzt sich immer zu der der Ventralgrenzrinne und der Grenzleiste fort, doch verdünnt sich ihre Dicke so, dass sie nur I/3-1/2 von der der betreffenden Schicht der Lippenplatte beträgt.

An der Lippenplatte verdickt sich die Tunica propria nicht nur polsterartig, sondern sie enthält auch einen hyalinen Knorpel. Trotz ihrem verwickelten Verlaufe können die Hauptbündel der fibrillären Bindegewebsfasern in dieser Schicht, wie Schumacher mitgeteilt hat, ihrem Verlauf nach in zwei Gruppen geteilt werden : die eine ist die tangentiale Faser; sie liegt teils subepithelial unter Zusammenschliessung in einer Schicht und umhüllt teils die submuköse Seite des hier auftretenden Knorpels als Knorpelhaut. Und zwar dehnt sich die letztere seitlich vom Knorpel aus und stellt die Grenze zwischen der Tunica propria und der Tunica submucosa dar. Die andere Gruppe ist die querverlaufende Faser, welche in die Tunica propria von aussen, nach innen hindurch unter Beimischung reicher, von der Umgebung eintretender Sehnenfaserbündel eintritt. Ihre Faser zeigt nicht nur das Aussehen, als ob sie aufgequollen wäre, sondern auch reiche feine Verästelung. Solche Ästchen verflechten sich miteinander oder mit den gröberen Bindegewebsfasern und bilden Netzwerke mit verschieden-grossen Maschen. Deswegen schrieb schon Schumacher, dass der Faserfilz der Lippenplatte in der tiefen Schicht, und bei den jungen Tieren einen Eindruck macht, der ähnlich dem des Gallertgewebes ist. Elastische Fasern sind fein und spärlich, und um die Bindegewebsfasern kaum erkennbar.

Bindegewebszellen scheinen nur vornehmlich in den Maschen zwischen den gröberen Bindegewebsfaserbündeln zerstreut umherzuliegen, dennoch können öfters 2-3 Zellen näher nebeneinander liegen. Meistens springen aus der Zelle einige schmale und lange Fortsätze hervor, durch welche wahrscheinlich die näher liegenden Zellen sich miteinander zu verbinden oder mit den Bindegewebsfasern der Umgebung im gewissen Zusammenhange zu stehen scheinen. Die Zelle ist meistens spindel- oder sternförmig, mehr voluminös als die anderen üblichen, und mit Farblösungen schwach gefärbt. Ihr Kern ist relativ gross und ovalförmig, der Chromatingehalt nicht ungemein reichlich.

Überhaupt tingiert sich dies Gewebe mit Hämatoxylin bläulich etwas 
stärker als die umgebenden Bindegewebe. Besonders färbt sich in einem Fall das benachbarte Gewebe um die Zelle höher blau, und zeigt es eigentlich auf den ersten Blick das Aussehen, als ob die Gewebe noch auf dem Wege zur Knorpelbildung seien. Die Zellen stellen sich etwas dichter mit Annäherung zum Knorpel auf, sie sind kugelig aufgequollen und zwar einige von der Knorpelkapsel umhüllt, doch zeigt ihr Kern keine bemerkbare Differenz von dem der obengenannten Bindegewebszellen. Zwischen den Zellen befinden sich aber wenige fibrilläre Bindegewebe, und die Zellen sind voneinander dadurch abgetrennt, deswegen bezeichnet sich dieses Gewebe als mit dem sogenannten Knorpeloidgewebe übereinstimmend. Dies geht meistens zum Knorpelgewebe ohne kenntliche Grenze über, doch sind stellenweise zwischen jenen und diesen feine Bindegewebsfaserbündelchen vorhanden.

Das Knorpelgewebe befindet sich in der Tiefe von 30-I 25 ". unter dem Epithel. Seine Dicke beträgt am Schnitt meist ca. 90-135/”, jedoch ist sie je nach den Stellen so verschieden, dass $45 \mu$ am dünnsten und $180 \%$ am dicksten ist. Und zwar zerlegt sich der Knorpel in den meisten Schnitten in zwei oder mehrere Stückchen, doch bietet er je nach dem Schnitte eine Platte dar, die den grösseren Teil der Breite der Tunica propria von der Lippenplatte einnimmt. Dessenungeachtet ist der Knorpel, betrachtet an den Rekonstruktionsmodellen, wie Figur 3 zeigt, eine ungleichgrosse vielfach durchlöcherte Platte von unregelmässiger Form. Diese Knorpelplatte liegt in der Mitte der Lippenplatte, und nach Berechnung am Rekonstruktions modell ist ihre Grösse nicht mehr als etwa $1 / 2$ von dem äusseren Durchmesser der LippenplatteI.6-1.9 $\mathrm{mm}$ lang und
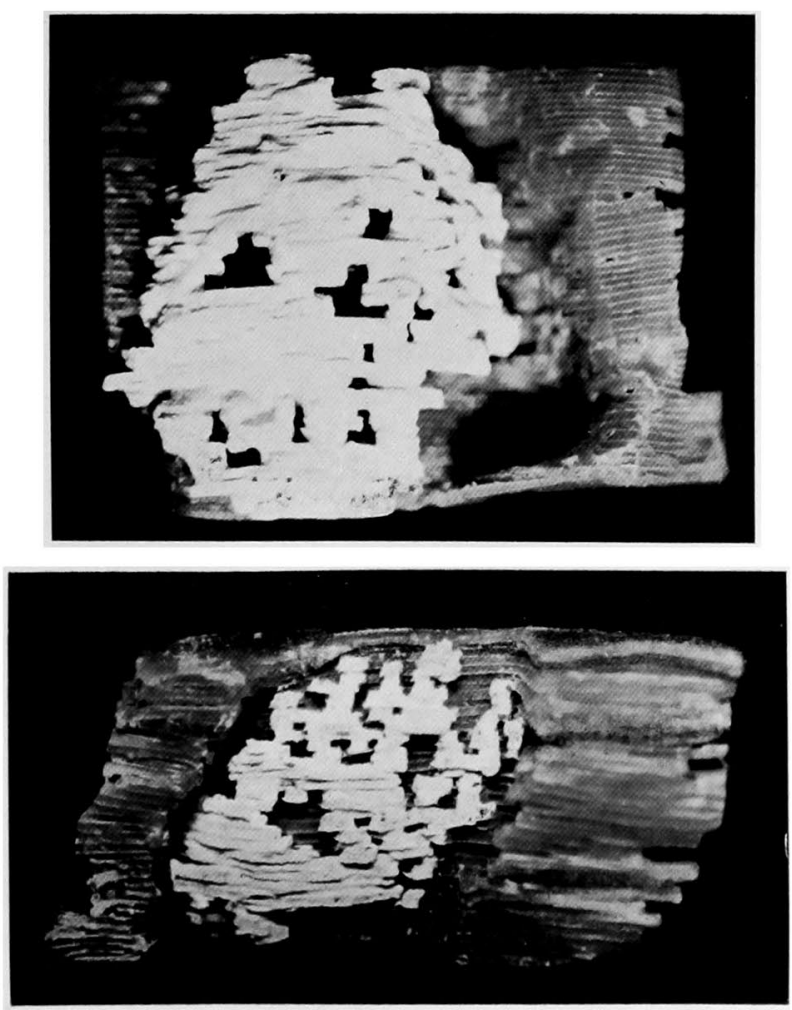

Fig. 3. Wachsmodelle der Lippenplatte (submuköse Fläche). 
0.8-I.6 mm breit. Die obengenannten Durchlöcherungen sind teils mit dem als Ausdehnung der Knorpelhaut ausgestreckten Bindegewebe ausgefüllt, teils führen sie die Blutkapillaren oder die von der Submucosa nach der Tunica propria ausstrahlend hineingetretenen Sehnenbündel.

Der Knorpel, was die Beschaffenheit der Zellen und der Grundsubstanz anbelangt, zeigt keine Differenz mit dem gewöhnlichen hyalinen Knorpel, doch ist die Knorpelhaut an seiner ganzen Umgebung in der Regel nicht erkennbar. Das Perichondrium umhüllt immer nur die submuköse Fläche des Knorpels und verdickt sich leichtgradig durch Eintritt der kurzen Sehnen der dort befindlichen Muskeln. Auf der übrigen Knorpelfläche ist nur stellenweise eine schwache, dünne, fibröse Haut sichtbar, welche eine Ausdehnung der Knorpelhaut darstellt und in diese übergeht, und auch, je nach dem Schnitte, scheint es, als ob sie die ganze Umgebung der Knorpel umhülle. Unter der Knorpelhaut sind die Knorpelzellen spindelförmig, wie man sie gewöhnlich sieht.

Um es kurz zu sagen, ist bei dem koreanischen Eichhorn (Eutamias asiaticus uthensis, Pallas) eine sogenannte Lippenplatte von Schumacher vorhanden. Sie stellt sich auf der Innenfläche der Oberlippe symmetrisch neben den beiden Seiten der Gaumenpapille ein und ist eine reiskorngrosse, im Vergleich mit der übrigen Mundschleimhaut mehr weisslich sichtbare, flache Erhebung. Nach der mikroskopischen Untersuchung weist man immer eine hyaline Knorpelplatte von unregelmässiger Form in der polsterartig verdickten Tunica propria nach.

Über die Bedeutung der Lippenplatte sind wir zwar zu gewissen Gedanken gelangt, aber wir behalten sie einem nachfolgenden Berichte vor. Nur möchten wir hier auf die Tatsache aufmerksammachen, dass es dicht hinter der Lippenplatte eine deutliche Falte gibt, die in die Backentasche tief nach unten verläuft und bei Betrachtung von der Mundhöhle spindelförmig ist.

\section{Literatur.}

Siegmund Schumacher: Eine ,Lippenplatte" beim Eichhornchen (Sciurus vulgaris L.). Anat. Anz. 58 Bd. S. $75-80.1924$. 